\title{
ROENTGENOGRAPHIC ANALYSIS OF LUMBAR LORDOTIC DIMENSIONS AMONG INDIGENES OF DELTA STATE
}

\author{
Ugochukwuka Ojieh, Abimbola Oladunni Ebeye* \\ *Department of Human Anatomy, Delta State University, Abraka, Nigeria. \\ Correspondence to Ugochukwuka Ojieh, Synergy Lifeline Consulting, Second Floor, Nicon Insurance \\ Plaza, Plot 242, Muhammadu Buhari Way, Central Business District, Abuja. Email: \\ ugoojieh@gmail.com, Phone: 08036988076.
}

\begin{abstract}
The spinal column of humans possesses strategically placed concave and convex curves which work in harmony to give an upright posture. The inwardly concave curvature in the lumbar region of the spine is known as the lordosis and it plays a very important role in the formation of the posture of humans. Analysis of the human lordosis is of great importance as it has been shown to be a major factor in the onset of low back pain, and is of great importance in spinal reconstructive surgery, through which some form of repair can be done to the spinal column that has undergone some form of alteration. The present study was carried out to analyse the lumbar lordotic dimensions among indigenes of Delta State. The aim of the study was to find out the average lordotic angle, vertebral body height and intervertebral disc height for the male and female population. The study was also aimed at finding the range for the normal lordotic angles as well as the correlations between these dimensions and the age of an individual. A regression equation was also formulated with which the lordotic angle of an individual can be estimated given the age of that individual. The sample size was 384 individuals (204 males and 180 females) with an age range of 20-60 years. The male population had a mean angle of $59.33 \pm 2.45^{\circ}$ and the females had a lower mean value of $53.33 \pm 2.61^{\circ}$. The study showed males had higher mean values for lumbar vertebra height and intervertebral disc height. A steady increase in the height of the lumbar vertebra and intervertebral disc from the first to the last was observed in this study. This study also showed that a weak negative correlation existed between the age of an individual and the lordotic angle and the vertebral height. Correlations between the age and intervertebral disc height showed a weak positive correlation. A regression formula was derived for estimating the lordotic angle of an individual given the age. This study will be of great importance to medical practitioners and forensic anthropologists in spinal reconstructive surgery and forensic human identification by gender and age.
\end{abstract}

Keywords: Lumbar Spine. Lordosis, Lordotic Angle, Correlation, Regression.

\section{INTRODUCTION}

Proper physique is important for optimal functionality of the human body. One of the major determining factors of the physique of an upright human is the structure of the spinal column (Farahani et al., 2012, Ambegaonkar et al., 2014, Been et al., 2014, Been et al., 2017). The upright posture of humans is constantly maintained by the spinal and pelvic muscles, hence weakness in this muscles, lack of proper exercise and inappropriate push or pull movements using these muscles will ultimately lead to body imbalances which culminate in improper posture (Kendall, 1983, Castillo \&
Lieberman, 2015).This upright posture in humans is as a result of the presence of characteristic concave and convex curves on the spinal column. The concave curves, known as Lordotic curves and are found in the cervical and lumbar regions of the spine while the convex curves, called Kyphotic curves and are found in the thoracic and sacral spinal regions. The presence of these curves in the spinal column of an upright man is necessary in the maintenance of the upright posture as it gives a great degree of stability to the head and torso over the lower limbs and allows for proper 
absorption of loads applied on the spinal column (Gracovetsky \& Iacono, 1987; Farfan, 1995; Gomez-Olivencia et al., 2013; Arsuaga et al., 2015). They also present him with the advantages of greater sight, increased speed and an increased gamut (Bushe-McGregor et al., 1981). Man can thus easily balance himself on two feet and use his upper limbs for functions other than locomotion.

One of the major postural analysis of an individual is that of his lumbar lordosis. Lumbar lordosis. Lumbar lordosis is defined to be the inwardly concave curvature which extends from the first lumbar vertebrae to the fifth lumbar vertebrae, formed majorly by the wedging of the intervetebral discs of each of these vertebrae and counters the forward inclination of the sacrum and thus restores the upward inclination of the spinal column. Of the five lumbar vertebrae which forms the lordosis, the last lumbar segment (L5) contributes almost $40 \%$ to overall lordosis while the first segment (L1) contributes only 5\% (Been et al., 2010).

Lumbar lordotic angle values for humans as reported by Been et al, (2013) ranges from $30^{\circ}$ to $80^{\circ}$. Values lower than this result in lumbar hypolordosis and values greater result in lumbar hyperlordosis. In the African and Nigerian perspective, studies on lumbar lordotic angles are evidently deficient and hence the knowledge of lumbar lordosis among African populations including Nigerians is sparse and those available, to the best of our knowledge have conflicting results (Patrick, 1976, Murrie et al., 2003, Legaye, 2007, Oyakhire et al., 2013). The general objective of this study was to study the lumbar lordotic dimensions among indigenes of Delta State.

\section{MATERIALS AND METHOD}

This study was a descriptive cross-sectional study and was retrospective in nature and radiographs which were used for this study were obtained from the archives of a selected tertiary health institution. The reasons for the retrospective nature of this study were among others to avoid the ethical issues of unnecessary exposure of individuals to radiation as some individuals, after radiologic evaluation would have presented pathological abnormalities and would be ultimately excluded from the study.

The heterogeneous population was stratified according to ethnic group into homogenous subsets of Delta State Indigenes. Elements were then randomly selected from each of the subsets in a manner which insured equal representation of each homogeneous subset.

For this study, 384 digital lateral lumbar radiographs of individuals between the ages of 18-60 years, were free of any congenital abnormalities and clearly showed that 5 lumbar vertebra and the intervertebral discs between L1 and L5 respectively where used. Radiographs taken with an Allengers Ceiling Suspended Static X-Ray Unit were displayed on a computer monitor screen so as to better highlight the landmarks from which the measurements were taken. Measurements were taken using the Clear Canvas (Version 3.0) software. The Cobb's method was used to measure the lumbar lordotic angle on the radiographs. The vertebral body height and the intervertebral disc height were measured using special digital vernier calipers on the Clear Canvas (Version 3.0) software.

Data collected from the study was subjected to statistical analysis using the IBM Corp, IBM SPSS Statistics for Windows, Version 22.0. Armonk, NY:IBM Corp. Results were analysed for Descriptive Statistics, Independent Sample T-test, Pearson Correlation and Regression equation. 


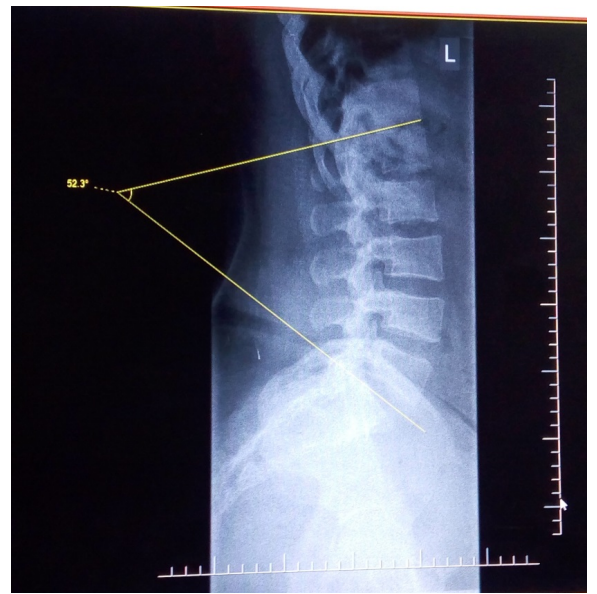

Figure 1. Measurement of Lumbar Lordotic angle

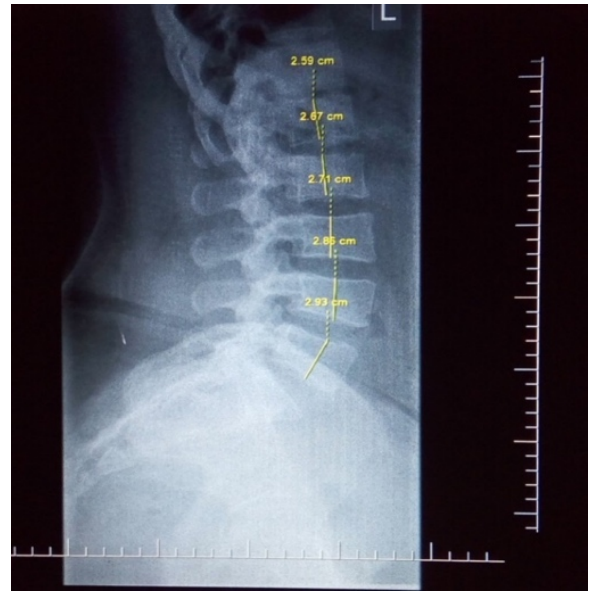

Figure 2. Measurement of Vertebral body height

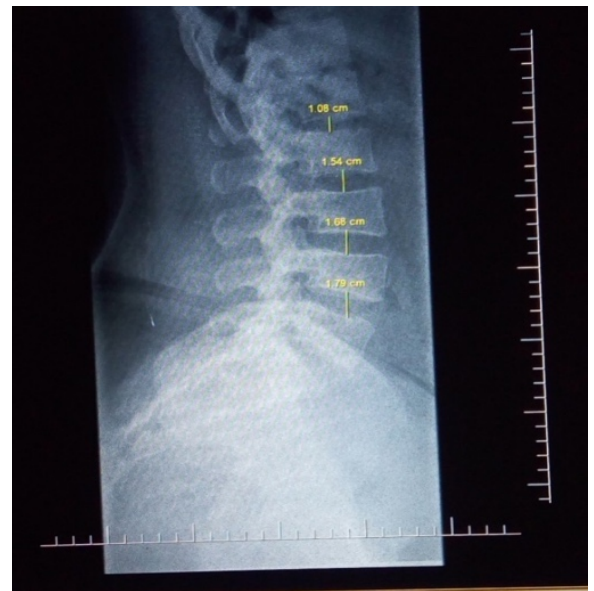

Figure 3. Measurement of Intervertebral disc height

\section{RESULTS}

Results from the study showed that males had a mean lumbar lordotic angle of $59.35^{\circ} \pm 2.45$ and females and mean lumbar lordotic angle of $53.33^{\circ} \pm 2.61$. Males thus showed higher mean values than females and this difference was shown to be statistically significant $(p=0.001)$. For the five lumbar vertebra, males had steady higher mean values than females. In every case

Table 1. Values for Male and Female Lumbar Lordotic Angles

\begin{tabular}{|l|l|}
\hline $\mathbf{N}=\mathbf{3 8 4}$ & VALUE \\
\hline MALE = 204 & $59.35^{\circ} \pm 2.45$ \\
\hline FEMALE = 180 & $53.33^{\circ} \pm 2.61$ \\
\hline $\mathbf{P}$ - Value & 0.001 \\
\hline
\end{tabular}

Table 2. Mean values for the five lumbar vertebrae in males and females

\begin{tabular}{|l|l|l|l|l|l|}
\hline & L1 & L2 & L3 & L4 & L5 \\
\hline MALE & $2.89 \pm 0.32$ & $3.05 \pm 0.29$ & $3.14 \pm 0.28$ & $3.25 \pm 0.26$ & $3.42 \pm 0.26$ \\
\hline FEMALE & $2.67 \pm 0.30$ & $2.84 \pm 0.28$ & $2.96 \pm 0.28$ & $3.09 \pm 0.28$ & $3.26 \pm 0.25$ \\
\hline P - Value & 0.001 & 0.001 & 0.001 & 0.001 & 0.001 \\
\hline
\end{tabular}




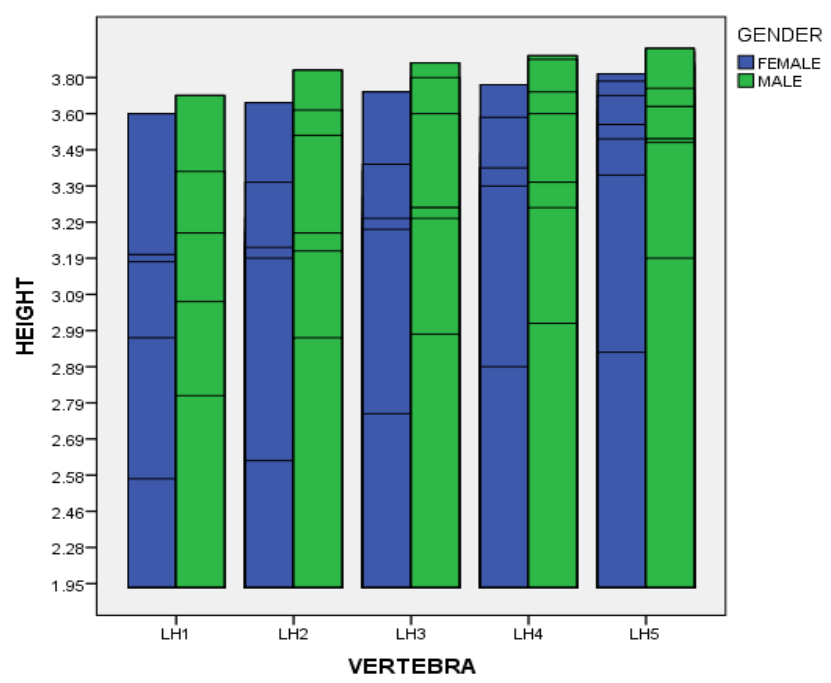

Figure 4. Graph showing the steady increase in mean values of the five lumbar vertebrae in males and females.

The four lumbar intervertebral discs also showed steady higher mean values in males than in females and these differences where all shown to be statistically significant $(p \leq 0.05)$;
(Table X). Among males and females, it was observed that the height of the intervertebral discs increased steadily from the first to the fourth intervertebral disc.

\begin{tabular}{|l|l|l|l|l|}
\hline & IVD1 & IVD2 & IVD3 & IVD4 \\
\hline MALE & $1.03 \pm 0.23$ & $1.23 \pm 0.25$ & $1.39 \pm 0.24$ & $1.56 \pm 0.26$ \\
\hline FEMALE & $0.97 \pm 0.22$ & $1.17 \pm 0.19$ & $1.32 \pm 0.18$ & $1.49 \pm 0.22$ \\
\hline P - Value & 0.033 & 0.001 & 0.002 & 0.005 \\
\hline
\end{tabular}

Table 3. Mean Values for the four intervertebral discs in males and females.

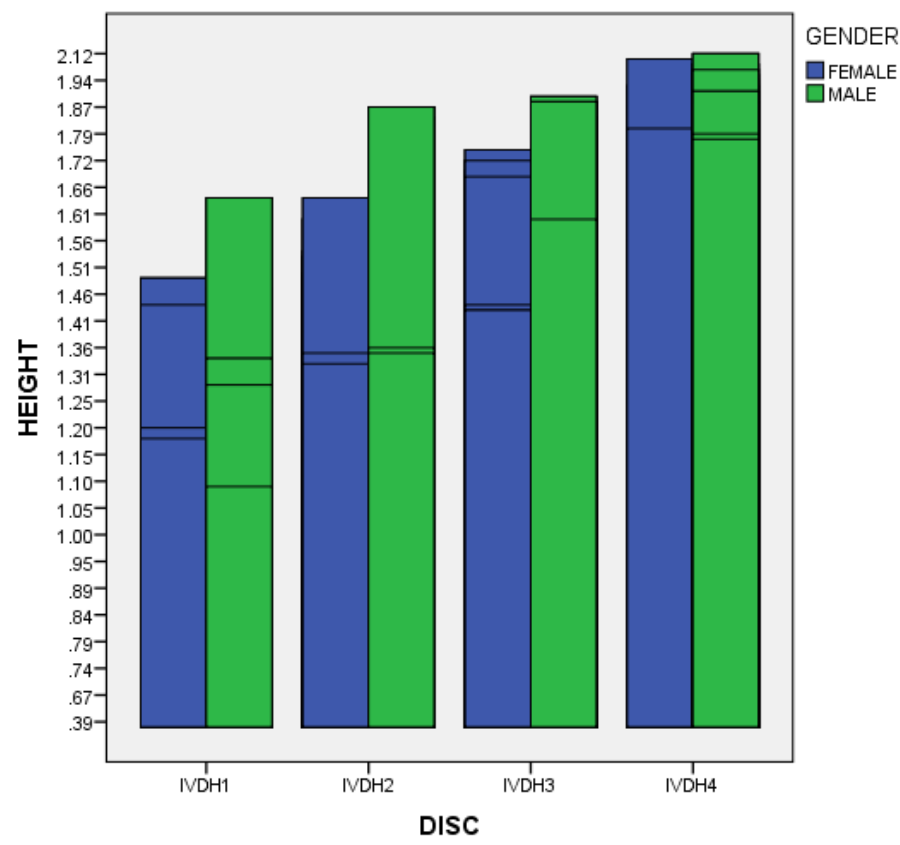

Figure 5: Graph showing the steady increase in intervertebral disc heights from the first to the fourth in males and females. 
Pearson Correlation tests showed that age had a weak negative correlation with lumbar lordotic angles and with vertebral body heights. This means that an increase in the age of an individual would lead to a decrease in the lumbar lordotic angle and lumbar vertebral body height. Conversely, the correlations between age and the lumbar intervertebral disc height were shown to be weak positive correlations. This means that an increase in the age of an individual would result in a corresponding increase in the lumbar intervertebral disc height. (Table X).

\begin{tabular}{|l|l|l|}
\hline & $\begin{array}{l}\text { Pearson Correlation } \\
\text { Coefficient }\end{array}$ & Sig. \\
\hline Age * LLA & -0.149 & 0.005 \\
\hline Age * L1 & -0.770 & 0.149 \\
\hline Age * L2 & -0.140 & 0.009 \\
\hline Age * L3 & -0.155 & 0.004 \\
\hline Age * L4 & -0.179 & 0.001 \\
\hline Age * L5 & -0.138 & 0.010 \\
\hline Age * IVD1 & 0.161 & 0.003 \\
\hline Age * IVD2 & 0.190 & 0.001 \\
\hline Age * IVD3 & 0.121 & 0.025 \\
\hline Age * IVD4 & 0.088 & 0.101 \\
\hline
\end{tabular}

Table 4. Correlations between age and various parameters

\begin{tabular}{|l|l|l|l|l|}
\hline \multicolumn{2}{|l|}{} & \multicolumn{2}{|l|}{ Female } & Male \\
\hline & Coefficient & Std. Error & Coefficient & Std. Error \\
\hline Constant (K) & 56.857 & .880 & 59.290 & .889 \\
\hline B & -.078 & .019 & .002 & .020 \\
\hline
\end{tabular}

Table 5. Regression table

The regression equation formula is given as $\mathrm{X}=\mathrm{k}+\mathrm{bY}$. Where; $\mathrm{X}=$ dependent variable. $\mathrm{k}=$ Constant; $\mathrm{b}=\ldots . . . ; \mathrm{Y}=$ independent variable; From the table 4.25 above; $k=56.857$ (females); 59.290 (males); $b=-0.078$ (females); 0.002 (males). Putting the figures above in the formula, the regression equation for LLA given the age of an individual is; Female $\mathbf{X}=$ $56.857-0.078 Y$. Male $X=59.290+0.002 Y$.

\section{DISCUSSION}

The study measured the lumbar lordotic angles of the males and females study population. The male participants had a minimum angle of $58.70^{\circ}$ and a maximum value of $65.80^{\circ}$, with a mean angle of $59.35 \pm 2.45^{\circ}$. Female participants had lower values with a minimum value of $39.40^{\circ}$ and a maximum value of $58.90^{\circ}$, with a mean angle of $53.33 \pm 2.61^{\circ}$. The results of this study varied from those of studies by Oyakhire et al., (2013) among indigenes of Port-Harcourt; Ayad et al., (2013) among Sudanese; Okpala (2014) in Southeastern Nigerians; Abdelmonem et al., (2014) in Egyptians, all of which showed 
greater lordotic angles values in Females compared to males. Several factors such as method of measurements and ethnicity could have been contributing factors to these variations.

The height of the five lumbar vertebrae which forms the lumbar lordosis was also measured in the course of this study (Tables 4.3- 4.7 and Figs. 4.3- 4.7). Among the male population the mean heights of the vertebrae were $2.89 \pm 0.32$ for $L 1,3.05 \pm 0.29$ for $L 2,3.14 \pm 0.28$ for $L 3$, $3.25 \pm 0.26$ for $L 4$ and $3.42 \pm 0.26$ for L5. The female population showed lower mean values for heights of the 5 lumbar vertebrae vis. $2.67 \pm 0.30$ for $L 1,2.84 \pm 0.28$ for $L 2,2.96 \pm 0.28$ for $L 3,3.09 \pm 0.28$ for $L 4$ and $3.26 \pm 0.25$ for $L 5$.

Among the male and female population a steady increase in the heights of the vertebrae was observed from L1-L5 with L5 being the largest of all (Fig. 4.8).These observation agreed with those of Busche-McGregor et al., (1981) who observed that the fifth lumbar vertebra was the largest and contributed most to the lumbar lordosis. A theory also accepted by Been and Kalichman, 2017.

The heights of the intervertebral discs located within the lumbar region of the spinal column were measured among the males and females with the males showing larger values for the heights compared to females (Tables 4.8 4.11 and Figs. 4-9-4.12).

Among the male population, the heights of the intervertebral discs were $1.03 \pm 0.23$, $1.23 \pm 0.25,1.39 \pm 0.24$ and $1.56 \pm 0.26$ for the first, second, third and fourth intervertebral discs respectively. Among the female population, the heights of the intervertebral discs were $0.97 \pm 0.22,1.17 \pm 0.19,1.32 \pm 0.18$ and $1.49 \pm 0.22$ for the first, second, third and fourth intervertebral discs respectively.

The values for the heights of the lumbar vertebra and their corresponding intervertebral discs showed that males had a longer lumbar lordosis than females, a theory that agreed with the study of Hay et al., (2015).

Independent samples T-test was used to test for significance of gender differences among all the variables. The mean difference was considered to be significant at a level of $\mathrm{P} \leq 0.05$.

The difference between the mean Lumbar Lordotic angle for males and females was shown to be statistically significant $(P=0.001)$. These results differed slightly from those of Oyakhire et al., (2013), Ayad et al, 2013, Okpala 2014 etc, who though found a statistically significant difference in lumbar lordotic angles of both genders, recorded females as having higher mean values for lumbar lordotic angles, compared to men.

For the five different lumbar vertebrae (L1-L5) males had larger mean values in all occasions and the mean difference between males and females, when tested, where all statistically significant $(L 1=0.001 ; L 2=0.001 ; \quad L 3=0.001$; $\mathrm{L} 4=0.001 ; \quad L 5=0.001)$. Males also had $a$ greater mean intervertebral disc height, compared to the females and the difference and the difference between the between the mean intervertebral disc height between males and females was statistically significant for all four lumbar intervertebral discs with $P$ values of $0.03,0.0,0.01$ and 0.05 for the first to fourth lumbar intervertebral respectively.

Pearson's correlation test carried out to find out the correlation existing between age and lumbar lordotic angle among the sample population showed that a weak negative correlation existed between both variables (0.149). This correlation was statistically significant $(P=0.005)$. This agreed with the study of Murrie et al., 2003 who stated that with increasing age there is a decrease of the lumbar lordotic angle. It however did not agree with the studies of Youdas et al., 1996 and Kalichman et al., 2011 who did not find any correlation between age and lumbar lordotic angle.

Results from this study also showed a negative correlation between age and the height of each of the five lumbar vertebra. This means that an increase in the age of an individual will ultimately lead to a decrease in the height of the lumbar vertebra.

Results however showed weak positive correlations between age and the height of the 
intervertebral disc among the individuals and in the case of the fourth lumbar intervertebral disc this correlation was statistically not significant. It may be hypothesized that the positive correlation noticed which means an increase in age will lead to an increase in intervertebral disc height is not unrelated to the negative correlation observed between age and vertebral height as a decrease in the vertebral height may be complimented by an increase in the corresponding intervertebral disc. This theory though, remains to be clarified.

A regression equation with which the lumbar lordotic angle of an individual can be calculated given the age of the individual was also developed. Where Female $X=56.857-0.078 Y$ and Male $X=59.290+0.002 Y$, where $X$ stands for the lumbar lordotic angle of the individual and $\mathrm{Y}$ stands for the age of the individual.

In conclusion indigenes of Delta State, males have a significantly longer lordosis and a higher lumbar lordotic angle than the females. This shorter and steeper curve noticed among the females could be attributed to various tasks which they undergo such as carrying of heavy loads on their heads, carrying of children on their backs and others which make for a much deeper curve in the lumbar spine of the women. This may also explain the higher incidence of low back pain in men than women as reported by Oyakhire et al., 2013 as the men are closer to having a flat back than the women. Unconfirmed reports in a study by Stagnara et al., 1982 have also reported that the steeper curve in women is as a result of the larger buttock size and more prominent backward curve in women compared to men. This occurrence brings about the need for a compensating forward lean of the lumbar region. Moore et al., 2014 also stated that the number of pregnancies a woman may have had could have an effect on her lumbar lordosis and he further stated that the new lordosis usually remains after pregnancy. This could also be hypothesized as a reason for the steeper lordosis among the females.

Results from this study showed that among indigenes of Delta State, normal lordosis ranges from $30^{\circ}-70^{\circ}$, a slight variation from the generally accepted range of $30^{\circ}-80^{\circ}$. This variation could be related to factors such as stature, occupation, and ethnicity.

It is recommended that this kind of research be carried out on more independent ethnic groups and if possible, using a prospective approach as this would give even more concise results.

\section{REFERENCES}

1. Abdelmonem, H. \& Raafat H., (2014). Midsagittal Anatomy of Lumbar Lordosis in Adult Egyptians: MRI Study. Anatomy Research International, Volume 2014 (2014), Article ID 370852, 1155/2014/370852.

2. Ambegaonkar, J.P., Caswell, S.V., Winchester, J.B. (2012). Upper-body muscular endurance in female university-level modern dancers: a pilot study. J Dance Med Sci. 16(1):3-7.

3. Arsuaga, J.L., Carretero, J.M., Lorenzo, C., Gomez-Olivencia, A., Pablos, A., Rodrıguez, L., Garcia-Gonzalez, R., Bonmatı, A., Quam, R.M., Pantoja-Perez, A (2015). Postcranial morphology of the middle Pleistocene humans from Sima de los Huesos, Spain. Proc Natl Acad Sci 112:11524-11529.

4. Ayad, C.E., Wahby, D.M.A., Abdalla, E.A.,\& AwadKajoak, S.(2013). Evaluation of Lumber Lordotic Angle in Patients with Inter Vertebral Disc Prolapse using Cobb's Method.Global Journal of Medical research Interdisciplinary, 13(7): 1-6

5. Been, E., Barash, A., Pessah, H.\& Peleg, S.(2010), A new look at the geometry of the lumbar spine, Spine, 35:1014-1017.

6. Been E., \& Kalichman L.(2013). Lordosis, The Spine Journal, Ocotber 2013, viewed 24 March 2016, https://www.researchgate.net/publication/257461591

7. Been, E., Gomez-Olivencia, A. \& Kramer, P.A.( 2014). Brief Communication: Lumbar lordosis in extinct hominins: Implications of the pelvic incidence. Am J Phys Anthropo/154:307-314. 
8. Been, E., Omez-Olivencia, E.A., Shefi, S., Soudack, M., Bastir, M., \& Barash A. (2017). Evolution of Spinopelvic Alignment in Hominins. The Anatomical Record 300:900-911

9. Busche-McGregor, M., Naiman, J.,\& Grice, A.S.(1981). Analysis of the lumbar lordosis in an asymptomatic population of young adults. The Journal of the CCA, 25(2):58-64

10. Castillo, E. R., \& Lieberman, D. E. (2015). Lower back pain. Evolution Medicine and Public Health, 1, 2-3.

11. Farfan, H.F.(1995). Form and function of the musculoskeletal system as revealed by mathematical analysis of the lumbar spine: an essay. Spine, 20:1462-1474

12. Farahani, A.H.S., Aghayari, A., Ghorbani, G.L (2012). The relation between body composition and spinal lordosis and kyphosis abnormalities in women. Journal of Research in Rehabilitation Sciences, 3:541-552

13. Gomez-Olivencia, A., Been, E., Arsuaga, J.L., Stock, J.T. (2013). The Neandertal vertebral column: 1-The cervical spine. J Hum Evol64: 608-630.

14. Gracovetsky, S.A.\& Iacono, S.(1987). Energy transfers in the spinal engine', J Biomed Eng,. 9:99-114

15. Hay, O., Dar, G., Abbas, J., Stein, D., May, H., \& Masharawi, Y. (2015) The Lumbar Lordosis in Males and Females, Revisited. PLoS ONE 10(8): e0133685.

https://doi.org/10.1371/journal.pone.0133685

16. Kalichman, L., Li, L., Hunter, D.J.\& Been, E.(2011). Association between computed tomography-evaluated lumbar lordosis and features of spinal degeneration, evaluated in supine position.Spine J, 11:308-315.

17. Kendall, F.M.E.(1983).Muscles Testing and Function, ( $3^{\text {rd } E d n), ~ W i l l i a m s ~ a n d ~ W i l k i n s: ~}$ Baltimore.

18. Moore, K.L., Dalley, A.F., Agur, A.M.R.(2014).Clinically Oriented Anatomy, (7th Edn,) Lippincott Williams \& Wilkins: Baltimore.

19. Murrie, V.L., Dixon, A.K., Hollinworth, W., Wilson, H.\& Doyle, T.A.C.(2003). Lumbar lordosis: Study of patients with and without low back pain. Clinical Anatomy. 16(2):144-147.

20. Okpala, F.O., (2014). Measurement of Lumbosacral Angle in Normal Radiographs: A Retrospective Study in Southeast Nigeria. Annals of medical and health sciences research. 4. 757-62. 10.4103/2141-9248.141548.

21. Oyakhire, M.O., Didia, B.C.\& Bob-Evans, Y.E.(2013). Radiographic evaluation of the Lumbar Lordotic angle of the Spine in a population of Nigerians', Asian journal of Medical science, 4(3): 69-75.

22. Patrick, J.M. (1976). Thoracic and lumbar spinal curvatures in Nigerian adults. Ann Hum Bioß:383-386.

23. Stagnara, P., De Mauroy, J.C., Dran, G., (1982). Reciprocal angulation of vertebral bodies in a sagittal plane: approach to references for the evaluation of kyphosis and lordosis. Spine, 7:335-342.

24. Youdas, J.W., Garrett, T.R.\& Harmsen, S.(1996). Lumbar lordosis and pelvic inclination of asymptomatic adults, Phys Ther, 76:1066-1081. 